\title{
缙云山亚热带森林林下常见薕类叶与细根分解 碳氮磷释放动态
}

杨术芳 ${ }^{1 \dagger}$, 豆鹏鹏 ${ }^{1 \dagger}$, 王红娟 ${ }^{2}$, 王芳 ${ }^{1}$, 杨光蓉 ${ }^{1}$, 林敦梅 ${ }^{*}$

1. 重庆大学三峡库区生态环境教育部重点实验室, 重庆 400045;

2. 重庆市农业科学院, 生物技术研究中心, 重庆 401329

$\dagger$ 同等贡献

*联系人, E-mail: lindunmei@cqu.edu.cn

2019-05-01 收稿, 2019-07-03 修回, 2019-07-04 接受, 2019-08-06 网络版发表

国家自然科学基金(31500356)、重庆大学中央高校基本科研业务费(2018CDXYCH0014)和重庆市技术创新与应用示范项目(重点研发项目) (cstc2018jszx-zdyfxmX0007)资助

\begin{abstract}
摘要 调落物分解是森林碳和养分循环的关键环节。长期以来森林调落物分解研究主要关注森林优势树种. 虽然 蒴类植物是亚热带森林林下层的重要组分, 占林下层大量的生物量, 但其调落物分解过程很少被关注. 本研究用缙 云山亚热带常绿阔叶林林下 12 种常见的苨类叶和细根进行分解实验, 监测分解 $113,198,386$ 和586 d后的碳氮磷释 放动态. 结果表明: 莿类叶与细根初始碳含量无显著差异, 但叶的初始氮磷含量均显著高于细根. 多数物种叶的碳 氮磷释放速率显著快于细根, 且叶的碳氮磷主要表现为简单的直接释放模式, 而细根的碳氮磷释放则表现出直接 释放、富集-释放、富集-释放-富集、始终富集等复杂模式. 氮、磷残余率随碳残余率的变化格局分别受初始氮、 磷含量的影响. 叶与细根间碳氮磷残余率的相关性表现出不同的格局: 碳残余率在分解 113,198 和 $386 \mathrm{~d}$ 后均为显著 正相关, 但 $586 \mathrm{~d}$ 后关系不显著; 而磷残余率除在 $113 \mathrm{~d}$ 时关系不显著外在其他时间点均显著正相关; 但氮残余率在 整个分解过程均无显著关系。该结果表明地上地下分解速率是否存在显著相关性与元素类型及分解时间有关. 本 研究结果为进一步量化蒴类植物对森林生态系统碳和养分循环的重要性提供参考.
\end{abstract}

关键词 䓲类植物, 叶分解, 细根分解, 养分释放, 化学计量学, 地上地下协变

调落物分解是影响碳循环和养分循环的重要生态 系统过程 ${ }^{[1-5]}$. 长期以来, 调落物分解研究主要以优势 种为对象, 而对森林的非优势种如林下层物种的关注 非常少. 近些年, 越来越多的研究表明林下层植物对森 林的结构和生态过程有重要影响, 包括植物群落的更 新以及生态系统碳和养分循环 ${ }^{[6 ~ 11]}$. 偋类植物是森林林 下层的重要组成部分, 贡献大量的物种和生物量 ${ }^{[11 ~ 15]}$, 在亚热带次生林或人工林中硕类植物常成为林下层的 优势物种 ${ }^{[13 ~ 16]}$. 蕨类植物在生态系统的碳和养分循环
方面也扮演着重要角色 ${ }^{[12]}$, 例如，Lyu等人 ${ }^{[16]}$ 发现亚热 带森林恢复过程中蕨类植物向土壤中输人了大量有机 碳, Marsh等人 ${ }^{[17]}$ 发现蕨类植物是磷和钾元素的重要储 藏库.

由于进化历史的差异 ${ }^{[18]}$, 莿类植物的生活史、解 剖结构及生理特征与种子植物存在较大的不同 ${ }^{[12]}$. 例 如，蕨类植物叶片的叶脉结构简单，组织少有次生生 长, 根系通常只有须根 ${ }^{[12]}$; 在温带落叶林中发现茨类植 物的比叶重、叶氮含量、叶绿素含量和最大光合速率 
都低于种子植物 ${ }^{[19]} ; \mathrm{Han}$ 等人 ${ }^{[20]}$ 分析了中国753种陆生 植物的叶片氮磷含量数据, 发现硕类植物叶片氮磷含 量低于种子植物; 偋类植物常含有更多的木质素、单 宁等物质 ${ }^{[21]}$. 烣类植物与种子植物间的差异性是否延 续到它们死亡残体的分解特性上, 即藏类植物与种子 植物调落物分解规律的异同性还有待于进一步研究. 有研究发现, 影响偋类植物与被子植物叶分解的关键 因子存在差异 ${ }^{[22]}$. 有些观点认为蕂类植物的分解速率 慢于种子植物 ${ }^{[21,23]}$, 但也有研究不支持此观点 ${ }^{[22,24]}$. 而 相比于大量的研究关注种子植物分解的养分释放动态 规律, 偋类分解的养分动态规律很少被报道.

土壤细菌和真菌通过分泌胞外酶驱动调落物的分 解及养分释放 ${ }^{[23,25]}$. 调落物基质对微生物来讲通常是 碳元素过量而氮和磷元素存在不足 ${ }^{[25,26]}$. 根据生态化 学计量学理论, 微生物分解者需要维持其生物量化学 元素比例(碳:氮:磷)处于相对稳定的状态 ${ }^{[26,27]}$. 微生物 常通过改变碳氮磷元素的利用效率或从周围环境吸 收、转运所缺乏的氮磷元素等方式来调节其化学计量 比值的稳定 ${ }^{[26,28]}$. 这些过程将会导致调落物在分解早 期出现氮磷元素富集的现象，随着分解的继续进行碳 氮比值和碳磷比值下降，当这些比值达到某一临界点 后将出现氮和磷元素的净释放 ${ }^{[2,3,28,29]}$. 这些过程主要 在种子植物凋落物分解中得到验证，而藓类分解过程 中氮磷元素的释放是否也存在类似的格局还缺乏相关 研究.

叶是最主要的地表凋落物, 而细根死亡残体是地 下调落物输人的最主要形式 ${ }^{[30]}$. 随着植物经济谱理论 的发展, 从整个植物体角度研究植物对生态过程的影 响是近些年的发展趋势 ${ }^{[31,32]}$. 越来越多的研究关注地 上地下调落物分解间的关联性 ${ }^{[33 \sim 35]}$. 如果叶和细根分 解存在协同变化, 那就意味着群落物种组成的改变将 同时、同方向地影响地上、地下相关生态过程; 此外, 地下分解过程也可以简单地使用地上分解过程进行预 测 ${ }^{[35]}$. 然而, 目前以种子植物为对象开展的叶与细根分 解关联性研究并没有统一结论. 例如, 在日本温带森林 中发现叶片和细根分解速率存在显著的正相关关 系 ${ }^{[34]}$, 但对欧洲和北美洲的温带树种开展的研究发现 两者无显著关系 ${ }^{[33]}$; 对中国亚热带和温带树种的叶片 和细根分解研究发现两者的相关性随分解的进行发生 改变 ${ }^{[35]}$. 蓱类植物类群中叶与细根分解是否存在协同 变化关系尚不清楚. 此外, 过去研究主要关注调落物质 量的变化, 而对地上地下调落物分解的元素释放是否
存在协同性很少涉及.

本研究以重庆缙云山亚热带常绿阔叶林林下 12 种 常见硕类的叶和细根为对象, 通过野外分解实验, 分析 偋类叶和细根的分解动态及碳氮磷元素的释放格局. 通常情况下植物叶的基质质量高于细根, 叶的分解速 率要快于细根 ${ }^{[31,36 ~ 40]}$, 基于已有研究结果, 我们预测 (1)：蕨类叶的分解及其养分释放速率要快于其细根; 根据生态化学计量比理论 ${ }^{[26,27]}$, 我们预测(2): 分解过程 中氮、磷残余率随碳残余率的动态变化格局将受初始 氮、磷含量的影响; 根据植物经济谱理论 ${ }^{[32]}$, 我们预测 (3): 在分解过程中蒴类叶和细根间的碳氮磷元素释放 存在协变关系.

\section{1 材料与方法}

\section{1 研究区概况}

本研究地点位于重庆市北碚区境内的缙云山国家 级自然保护区 $\left(106^{\circ} 17^{\prime} 43^{\prime \prime} \sim 106^{\circ} 24^{\prime} 50^{\prime \prime} \mathrm{E}, 29^{\circ} 41^{\prime} 08^{\prime \prime}\right.$ $\left.29^{\circ} 41^{\prime} 03^{\prime \prime} \mathrm{N}\right)$. 海拔350 951.5 m, 气候类型为亚热带季 风气候, 年平均气温 $13.6^{\circ} \mathrm{C}$, 年均降水量 $1610 \mathrm{~mm}$, 相 对湿度 $87 \%$; 土壤类型主要是酸性黄壤; 植被类型以典 型的中亚热带常绿阔叶林为主，常见乔木树种包括栲 (Castanopsis fargesii)、润楠(Machilus pingii)、四川山 矾(Symplocos setchuensis)、薯豆(Elaeocarpus japonicus)、四川大头茶(Gordonia acuminata) 和马尾松(Pinus massoniana $)$ 等 ${ }^{[41]}$. 蓱类植物是该区域亚热带常绿阔叶 林林下层植物的重要组成部分, 共有偋类植物 38 科 74

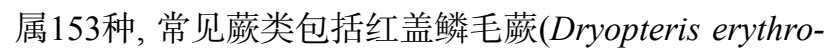
sora)、中华复叶耳硕(Arachniodes chinensis)、狗脊 (Woodwardia japonica)、边缘鳞盖硕(Microlepia marginata $)$ 和中华里白(Diplopterygium chinense $)^{\text {等 }}{ }^{[42]}$.

\section{2 䕇类样品采集}

本研究选择缙云山常绿阔叶林林下12种常见偋类 植物 (表 1 )的叶和细根(直径 $\leqslant 2 \mathrm{~mm}$ ) 为分解实验材料. 由于细根的死亡时间很难确定，因此参照已有研究的 方法 ${ }^{[43]}$, 我们采集活的细根和叶片进行分解实验. 薕类 叶与细根材料于 2016年10 12月期间在缙云山保护区 内收集. 在剪下薕类植物的成熟叶片后用铁铲挖出其 全部根系并用清水清洗干净. 每个物种的叶和细根采 集自20 50株成熟鰀类植物个体. 将叶和细根置于 $40^{\circ} \mathrm{C}$ 烘箱中干燥 $48 \mathrm{~h}$, 充分混合后装人自封袋中放置在黑暗 
表 1 莿类叶与细根初始碳氮磷元素含量 ${ }^{a)}$

Table 1 Initial leaf and fine root carbon, nitrogen and phosphorus concentrations of the 12 fern species

\begin{tabular}{|c|c|c|c|c|c|c|c|}
\hline \multirow{2}{*}{ 物种 } & \multirow{2}{*}{ 拉丁名 } & \multicolumn{2}{|c|}{ 碳含量(mg/g) } & \multicolumn{2}{|c|}{ 氮含量(mg/g) } & \multicolumn{2}{|c|}{ 磷含量(mg/g) } \\
\hline & & 叶 & 细根 & 叶 & 细根 & 叶 & 细根 \\
\hline 薄盖双盖硕 & Diplazium hachijoense & $396.57 \pm 13.01$ & $398.66 \pm 10.32$ & $23.54 \pm 0.07$ & $6.33 \pm 0.20$ & $2.51 \pm 0.17$ & $1.84 \pm 0.059$ \\
\hline 齿果鳞毛菜 & Dryopteris labordei & $432.38 \pm 9.79$ & $423.10 \pm 8.13$ & $22.25 \pm 0.17$ & $9.94 \pm 0.15$ & $2.44 \pm 0.06$ & $1.36 \pm 0.017$ \\
\hline 翅轴蹄盖硕 & Athyrium delavayi & $397.47 \pm 6.97$ & $410.39 \pm 2.57$ & $21.78 \pm 0.46$ & $10.77 \pm 0.31$ & $2.64 \pm 0.03$ & $1.29 \pm 0.004$ \\
\hline 狗脊 & Woodwardia japonica & $403.13 \pm 8.17$ & $414.56 \pm 4.55$ & $16.03 \pm 0.10$ & $5.11 \pm 0.05$ & $1.29 \pm 0.03$ & $0.55 \pm 0.013$ \\
\hline 红盖鳞毛硕 & Dryopteris erythrosora & $428.92 \pm 6.79$ & $425.02 \pm 10.92$ & $19.41 \pm 0.29$ & $6.23 \pm 0.17$ & $1.38 \pm 0.04$ & $0.80 \pm 0.009$ \\
\hline 毛柄双盖茨 & Diplazium dilatatum & $363.19 \pm 6.54$ & $375.78 \pm 17.28$ & $25.25 \pm 0.21$ & $14.93 \pm 0.12$ & $1.95 \pm 0.04$ & $1.44 \pm 0.090$ \\
\hline 披针新月硕 & Pronephrium penangianum & $403.74 \pm 10.19$ & $396.03 \pm 5.34$ & $21.32 \pm 0.12$ & $9.73 \pm 0.24$ & $2.81 \pm 0.10$ & $0.93 \pm 0.011$ \\
\hline 疏羽凸轴偋 & Metathelypteris laxa & $413.37 \pm 6.56$ & $404.23 \pm 6.61$ & $19.59 \pm 0.13$ & $7.9 \pm 0.14$ & $1.52 \pm 0.10$ & $1.03 \pm 0.032$ \\
\hline 乌嵚 & Odontosoria chinensis & $422.84 \pm 3.83$ & $382.34 \pm 9.97$ & $19.66 \pm 0.28$ & $8.48 \pm 0.14$ & $1.53 \pm 0.02$ & $0.57 \pm 0.024$ \\
\hline 中华复叶耳濒 & Arachniodes chinensis & $418.96 \pm 5.22$ & $436.86 \pm 6.43$ & $17.72 \pm 0.34$ & $11.06 \pm 0.43$ & $1.10 \pm 0.02$ & $0.47 \pm 0.012$ \\
\hline 中华里白 & Diplopterygium chinense & $426.16 \pm 12.55$ & $425.56 \pm 7.03$ & $20.21 \pm 0.08$ & $8.66 \pm 0.08$ & $0.86 \pm 0.02$ & $0.50 \pm 0.006$ \\
\hline 紫萁 & Osmunda japonica & $410.49 \pm 10.90$ & $413.63 \pm 4.09$ & $16.42 \pm 0.12$ & $14.87 \pm 0.40$ & $1.32 \pm 0.06$ & $0.78 \pm 0.003$ \\
\hline 变异系数 & & $5 \%$ & $4 \%$ & $14 \%$ & $33 \%$ & $37 \%$ & $45 \%$ \\
\hline 所有物种均值 & & $409.77 \pm 5.50^{\mathrm{a}}$ & $408.85 \pm 5.27^{\mathrm{a}}$ & $20.27 \pm 0.80^{\mathrm{a}}$ & $9.5 \pm 0.90^{\mathrm{b}}$ & $1.78 \pm 0.19^{\mathrm{a}}$ & $0.96 \pm 0.13^{\mathrm{b}}$ \\
\hline
\end{tabular}

a) 数据为均值 \pm 标准误. 数据后面不同小写字母代表统计上差异显著 $(P<0.05$; 配对 $t$ 检验 $)$

处备用. 由于分解后回收的样品进行的是 $65^{\circ} \mathrm{C}$ 烘干处 理, 因此我们分别称取每种蕨类的叶片和细根样品各 3 份, 先后在 40 和 $65^{\circ} \mathrm{C}$ 烘箱中干燥 $48 \mathrm{~h}$ 后称重, 计算 $65^{\circ} \mathrm{C}$ 烘干和 $40^{\circ} \mathrm{C}$ 烘干处理间的质量比值，该比值即为不同 温度烘干间的质量转换系数 ${ }^{[4]}$. 每种偋类的叶片和细 根样品各随机选3份用球磨机进行粉碎, 然后测定未分 解状态下的叶片和细根的碳氮磷含量(浓度). 碳含量用 岛津TOC-TN分析仪配上固体进样器进行直接测定; 氮 和磷含量测定前先用 $98 \%$ 的 $\mathrm{H}_{2} \mathrm{SO}_{4}$ 和 $30 \%$ 的 $\mathrm{H}_{2} \mathrm{O}_{2}$ 将样 品在高温下进行充分消解, 然后用岛津TOC-TN分析仪 测定氮含量, 用钼锑钪分光光度法测定磷含量.

\section{3 原位分解实验}

本研究使用分解袋法进行分解实验. 分解袋大小 为 $15 \mathrm{~cm} \times 10 \mathrm{~cm}$, 孔径为 $25 \mu \mathrm{m}$, 每个分解袋装人 $3 \mathrm{~g}$ 叶 或 $2 \mathrm{~g}$ 细根, 并挂上塑料号码牌进行标记. 制作的分解袋 的数量为: 2 种分解材料 $\times 12$ 个物种 $\times 4$ 次回收 $\times 3$ 个分解 场地 $\times$ 每个分解场地每次回收 2 个重复, 共计 576 个. 3 个 分解场地间的直线距离大约为 $200 \mathrm{~m}$, 均位于缙云山常 绿阔叶林林下较平坦的区域. 分解袋于 2017 年1月25日 布设. 布设分解袋时, 先去除地表新鲜凋落物, 然后用 塑料钉将装有叶片的分解袋固定在地表, 保证分解袋
之间不重叠, 并将原地凋落物放回分解袋间的间隙; 将 细根分解袋垂直埋在5 10 cm深度的土壤中, 并覆盖原 有的调落物. 由于凋落物在早期分解快, 后期分解慢, 因此分解袋按早期回收时间间隔短，后期回收时间间 隔长进行回收, 共计4次回收, 回收时间分别为: 2017年 5 月 1 日(113 d)、2017年7月24日(198d)、2018年1月26 日 $(386 \mathrm{~d})$ 和 2018 年 8 月 12 日 $(586 \mathrm{~d})$. 每次回收后将分解 袋中剩余的材料取出, 如有其他杂质先将其去除, 然后 置于 $65^{\circ} \mathrm{C}$ 烘箱中干燥 $48 \mathrm{~h}$ 并称重. 从每种材料每次回收 中的 6 个重复中选择 3 份(每个分解场地各 1 份)用球磨机 进行粉碎, 用于测定碳、氮和磷元素的含量. 本研究仅 使用测定了元素含量的分解袋数据进行后续分析. 最 终, 质量残余率用公式(1)进行计算, 碳氮磷元素的残 余率用公式(2)进行计算:

$$
\begin{aligned}
& M_{\mathrm{r}}=\left(M_{\mathrm{f}}\right) /\left(M_{\mathrm{i}} \times C F\right), \\
& E_{\mathrm{r}}=\left(M_{\mathrm{f}} \times E_{\mathrm{f}}\right) /\left(M_{\mathrm{i}} \times C F \times E_{\mathrm{i}}\right) \times 100 \%,
\end{aligned}
$$

式中 $M_{\mathrm{r}}$ 代表质量残余率; $E_{\mathrm{r}}$ 代表碳、氮或磷的残余率; $M_{\mathrm{i}}$ 代表调落物的初始质量 $\left(40^{\circ} \mathrm{C}\right.$ 烘干), $M_{\mathrm{f}}$ 代表调落物 分解后的质量 $\left(65^{\circ} \mathrm{C}\right.$ 烘干); $C F$ 为 $40^{\circ} \mathrm{C}$ 烘干与 $65^{\circ} \mathrm{C}$ 烘干 间的质量转换系数; $E_{\mathrm{i}}$ 和 $E_{\mathrm{f}}$ 分别代表分解前后碳、氮或 磷元素的含量. 


\section{4 统计分析}

首先对数据进行正态性和方差齐性检验. 用配对 样本 $t$ 检验(paired $t$-test)分析萨类叶与细根间的初始 碳、氮、磷含量是否存在显著差异. 用双因素方差分 析(two-ways ANOVA)检验不同物种和不同器官在经 过 $586 \mathrm{~d}$ 分解后的质量损失以及碳氮磷的释放量是否存 在显著差异, 用Tukey检验进行多重比较分析. 为检验 分解过程中氮、磷残余率随碳残余率的动态变化格局 是否因初始氮、磷含量的不同而存在差异，将所有数 据点整合，根据分解材料初始氮或磷含量大小划分成 低氮(初始氮 $<8.5 \mathrm{mg} / \mathrm{g})$ 、中等氮 $(8.5 \mathrm{mg} / \mathrm{g}<$ 初始氮 $<21 \mathrm{mg} / \mathrm{g}$ ) 和高氮(初始氮 $>21 \mathrm{mg} / \mathrm{g}$ ), 以及低磷(初始磷 $<0.8 \mathrm{mg} / \mathrm{g})$ 、中等磷 $(0.8 \mathrm{mg} / \mathrm{g}<$ 初始磷 $<1.9 \mathrm{mg} / \mathrm{g})$ 和高 磷(初始磷 $>1.9 \mathrm{mg} / \mathrm{g}$ ) 共计6组数据. 然后用 “ $\mathrm{mgcv}$ ”程序 包中的广义加性模型(Generalized Additive Models, $\mathrm{GAM})^{[44]}$ 分别分析低、中、高初始氮3组数据的氮残余 率随碳元素释放的动态变化格局，以及低、中、高初 始磷 3 组数据的磷残余率随碳元素释放的动态变化格 局. 为探讨蒝类叶与细根分解间的协变关系及此关系 随分解时间的变化格局, 我们用标准化主轴回归(standardized major axis regression, SMA) ${ }^{[45]}$ 分别分析不同 时间段回收的叶与细根之间碳氮磷残余率的相关性. 所有统计分析在 $\mathrm{R}$ version 3.5.1中进行, $P<0.05$ 定为统 计上显著.

\section{2 结果与分析}

\section{1 蕨类叶与细根初始碳氮磷含量}

茨类植物叶与细根的初始碳含量变化范围在 363.19 436.86 mg/g之间, 不同物种间的变异性较小, 叶 和细根的变异系数仅为 $5 \%$ 和 $4 \%$ (表 1$)$, 且叶与细根之间 的初始碳含量无显著差异 $(P=0.84)$. 与初始碳含量的格 局不同，叶的初始氮及磷含量均显著大于细根(两者 $P<0.001)$. 叶和细根初始氮含量的变异性较大, 其变异 系数分别为 $14 \%$ 和 $33 \%$ (表1). 叶和细根初始氮含量最大 的均是毛柄双盖蒴(叶: $25.25 \pm 0.21 \mathrm{mg} / \mathrm{g}$; 细根: $14.93 \pm$ $0.12 \mathrm{mg} / \mathrm{g}$ ), 分别是初始氮含量均最小的狗脊(叶: $16.03 \pm 0.10 \mathrm{mg} / \mathrm{g}$; 细根: $5.11 \pm 0.05 \mathrm{mg} / \mathrm{g}$ )的 1.58 和 2.92 倍 (表1). 与氮类似, 叶和细根的初始磷含量变异系数达到 $37 \%$ 和 $45 \%$ (表1). 翅轴蹄盖蓱的叶初始磷含量最大 $(2.64 \pm 0.03 \mathrm{mg} / \mathrm{g})$, 是叶磷含量最小的中华里白 $(0.86 \pm$
$0.02 \mathrm{mg} / \mathrm{g}$ )的 3.07 倍，而薄盖双盖偋的细根初始磷含量 最大 $(1.84 \pm 0.059 \mathrm{mg} / \mathrm{g})$, 是细根磷含量最小的中华里白 $(0.50 \pm 0.006 \mathrm{mg} / \mathrm{g})$ 的 3.68 倍(表1).

\section{2 叶与细根元素释放动态}

经过 $586 \mathrm{~d}$ 的野外分解，12种鄀类叶与细根间的质 量及碳氮磷元素的残余率均存在显著差异 (所有 $P<0.001$; 图1). 披针新月硕的叶质量及碳氮磷的残余率 均最少, 分别为 $25.92 \%, 29.96 \%, 25.54 \%$ 和 $12.64 \%$, 而 中华里白均最多，分别为 $57.95 \%, 55.70 \%, 70.71 \%$ 和 94.02\% (图1); 对细根来讲, 翅轴蹄盖萍的碳、紫萁的 氮和毛柄双盖䓲的磷残余率最少，分别为 $42.69 \%$, $40.67 \%$ 和 $23.73 \%$ ，而中华里白的碳残余率、红盖鳞毛 濒的氮残余率和中华复叶耳蒴的磷残余率分别最大, 多达 $85.22 \% ， 149.64 \%$ 和 $123.50 \%$ 。除紫萁、疏羽凸轴

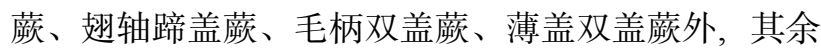
物种叶质量和碳残余均显著小于细根(图1(a)，(b)); 除 紫萁、中华里白和中华复叶耳硕外，其余物种叶的氮 残余率均显著小于细根(图1(c)); 中华复叶耳硕、狗

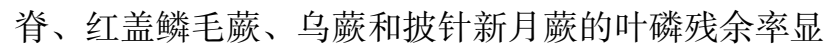
著小于细根, 其余物种差异不显著(图1(d)).

从整个分解过程 $(0 \sim 586 \mathrm{~d})$ 来看, 所有物种叶的碳 元素及大部分物种叶的氮磷元素释放表现为直接释放 模式，而细根的元素释放模式更为复杂(图2 4). 例如,

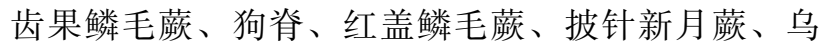
蕨、中华复叶耳硕、中华里白的细根在分解早期碳元 素几乎没有释放，甚至出现少量增加(图2); 薄盖双盖 莿、狗脊及红盖鳞毛蒝的细根氮元素在0 586 d的分解 过程中始终处于富集状态，而齿果鳞毛蕨和疏羽凸轴 萨则表现为先富集后释放，其余物种表现为直接释放 (图3); 狗脊细根的磷元素表现为富集-释放-再富集, 而 中华复叶耳萨则表现为先释放后富集，其余物种表现 为直接释放(图4).

氮残余率与碳残余率的相关性分析表明不同初始 氮含量的数据组内氮残余率与碳残余率之间均呈现显 著的、近似于单峰曲线的关系，即随着碳残余率的减 少, 氮残余率先上升后下降(图5(a) (c)). 但不同初始氮 数据组间的曲线最高点存在差异, 在低氮数据组, 曲线 最高点高于 $100 \%$, 即氮是富集的状态(图5(a)), 而在 中、高氮数据组，最高点都低于 $100 \%$, 即已处于氮释 放的状态(图5(b), (c)). 对低初始磷数据组的分析发现, 磷残余率与碳残余率无显著的关系(图5(d)), 但在去除 1 

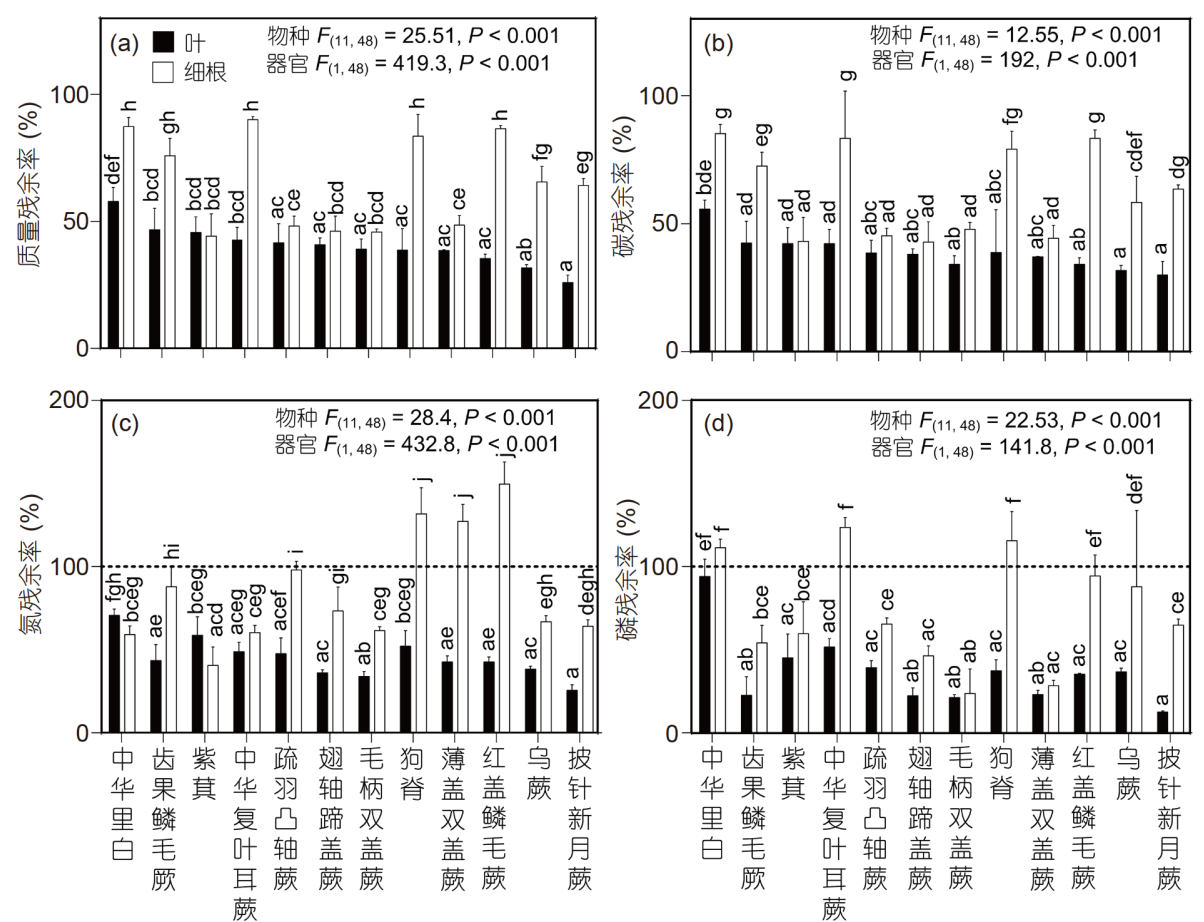

图 1 蔂类叶与细根经过 $586 \mathrm{~d}$ 分解后的质量及碳氮磷元素的残余率. 数据为均值土标准误 $(n=3)$, 条形图上没有相同字母表示统计上差异显著 $(P<0.05)$

Figure 1 Fern leaf and root mass loss, carbon, nitrogen and phosphorus remains after $586 \mathrm{~d}$ field decomposition. Data are mean \pm SE $(n=3)$. No identical lowercase letters in the bar indicated significant differences $(P<0.05)$

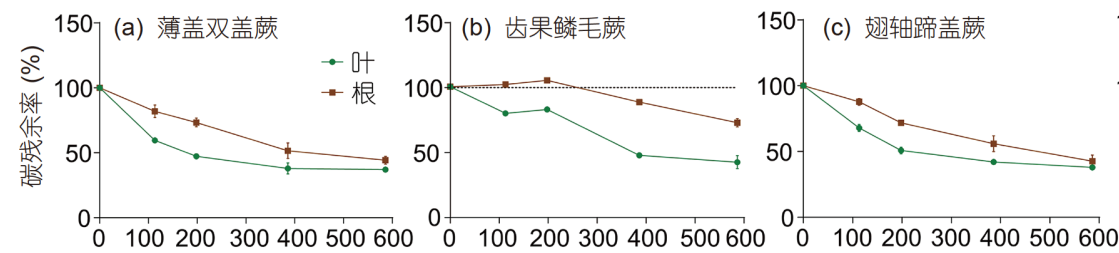

] (d) 狗脊
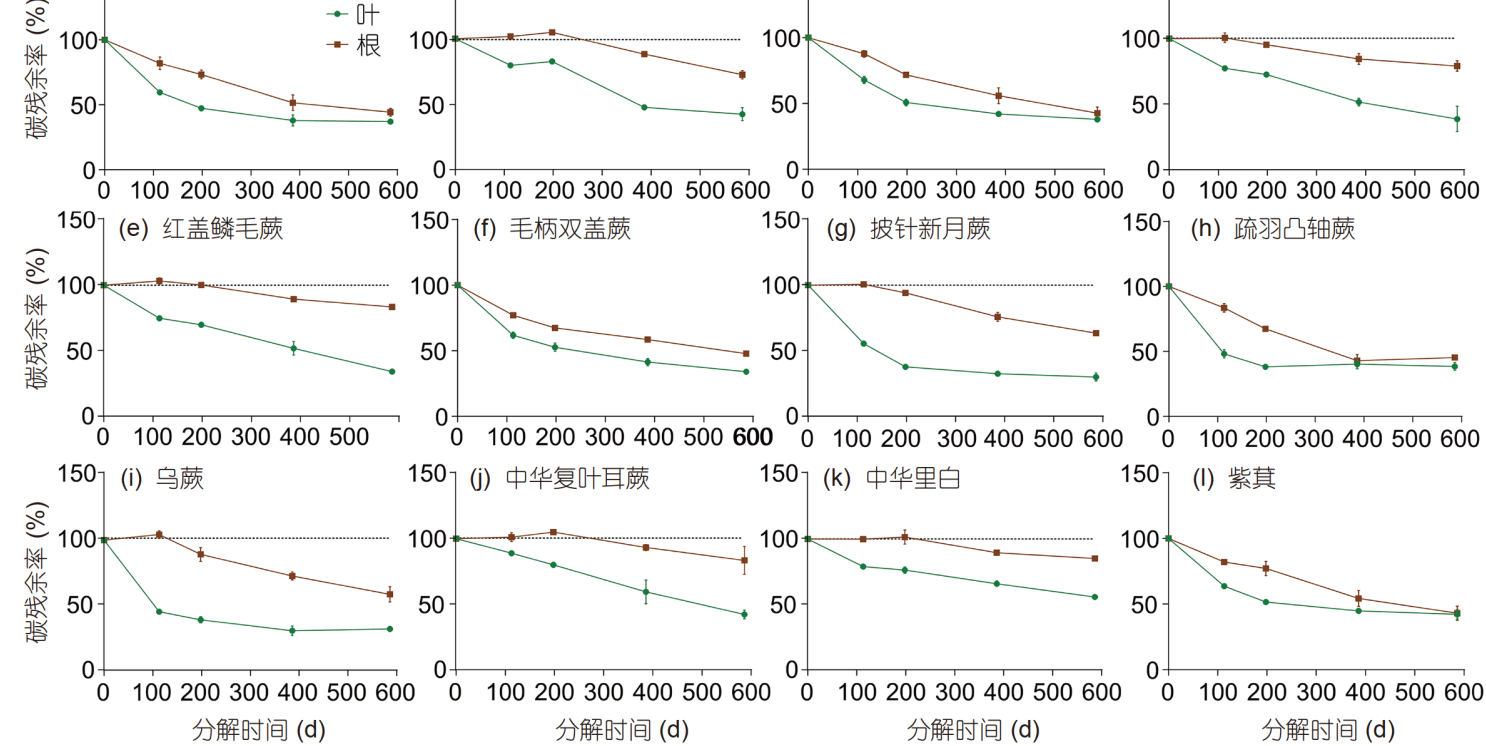

图 2 (网络版彩色) 濒类植物叶和细根在 $586 \mathrm{~d}$ 分解过程中碳元素的动态变化. 每个数据点为均值 \pm 标准误 $(n=3)$

Figure 2 (Color online) Carbon dynamics of fern leaves and fine roots during the $586 \mathrm{~d}$ field decomposition. Points are mean $\pm \mathrm{SE}(n=3)$

个可能存在异常的数据点后两者呈现显著的正相关关 系 $\left(y=0.18 x-7.79, R^{2}=0.12, P<0.01\right)$, 即对低初始磷的数 据组, 随着分解的进行细根始终处于不断富集磷元素
的状态. 而在中、高初始磷数据组内磷残余率与碳残 余率之间均呈现显著的单峰曲线关系，即与氮元素类 似, 随着碳残余率的减少, 磷残余率先上升后下降(图5 

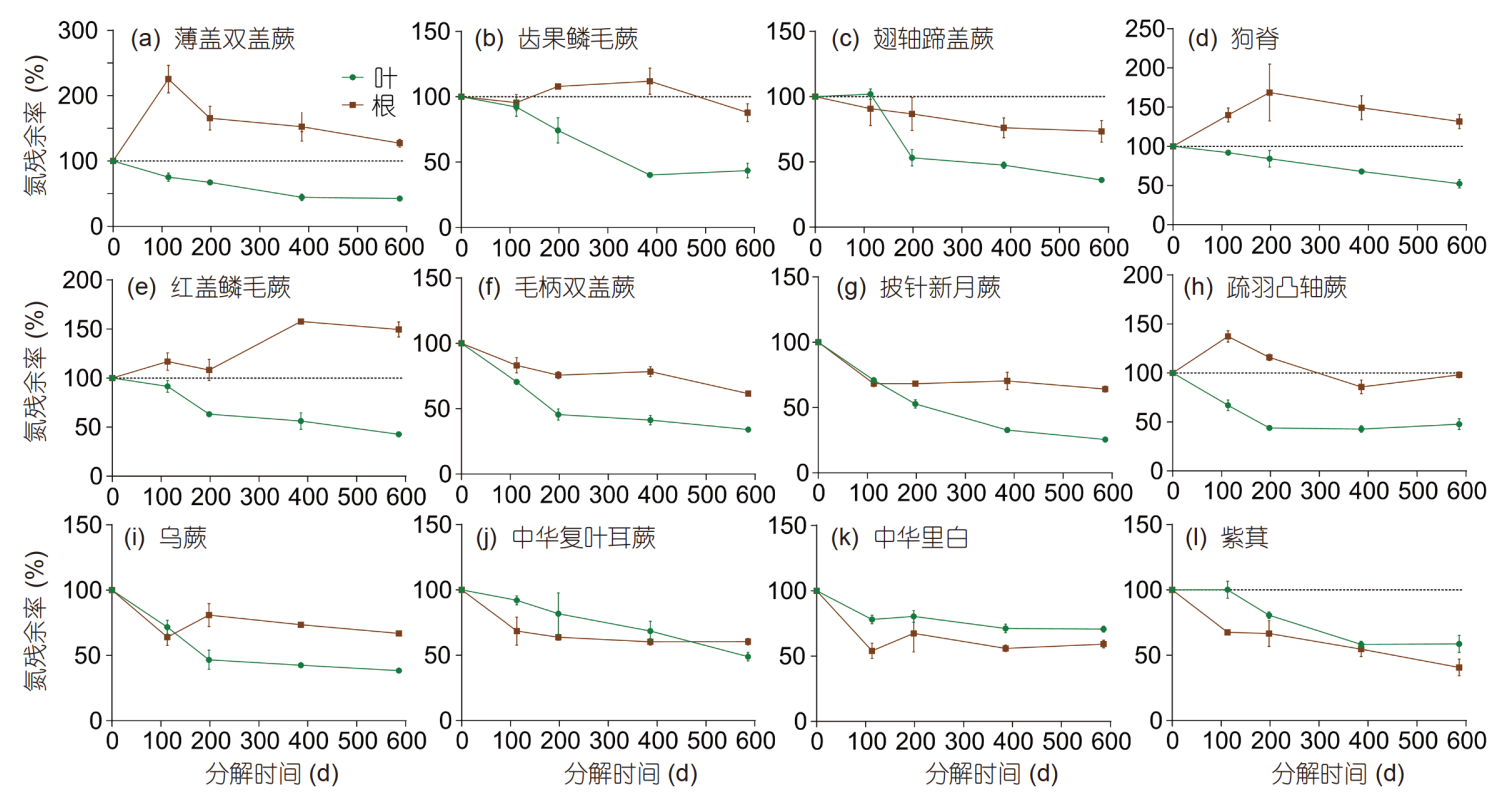

图 3 (网络版彩色)藏类植物叶和细根在 $586 \mathrm{~d}$ 分解过程中氮元素的动态变化. 每个数据点为均值土标准误 $(n=3)$

Figure 3 (Color online) Nitrogen dynamics of fern leaves and fine roots during the $586 \mathrm{~d}$ field decomposition. Points are mean $\pm \mathrm{SE}(n=3)$
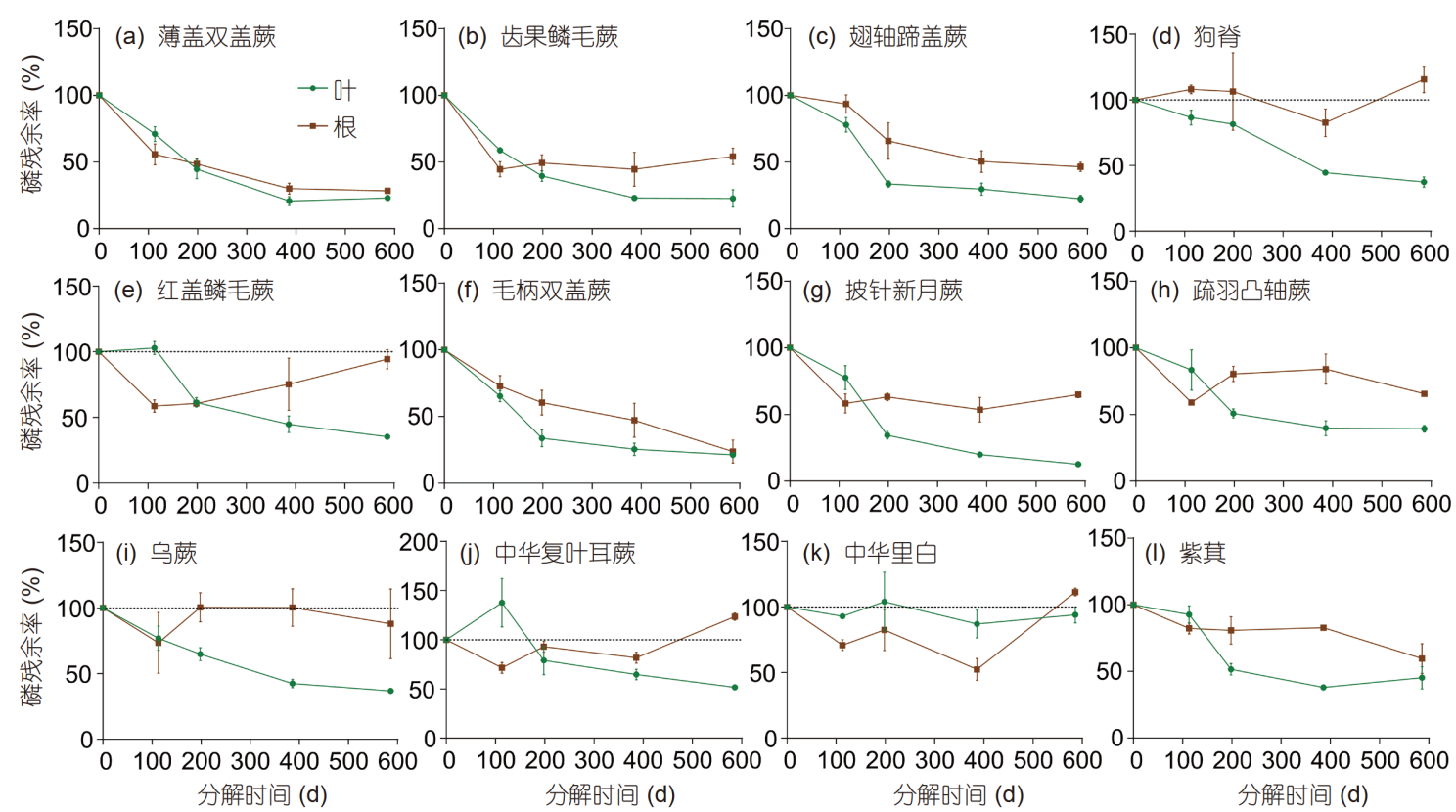

图 4 (网络版彩色) 莎类植物叶和细根在 $586 \mathrm{~d}$ 分解过程中磷元素动态变化. 每个数据点为均值 \pm 标准误 $(n=3)$

Figure 4 (Color online) Phosphorus dynamics of fern leaves and fine roots during the $586 \mathrm{~d}$ field decomposition. Points are mean \pm SE $(n=3)$

(e) (f)).

\section{3 地上叶与地下细根间碳氮磷元素释放的相关性}

地上叶与地下细根间元素的释放格局仅部分表现 出相关性, 且随分解时间段的不同发生改变(图6). 叶与
细根间碳元素的残留率在早期的 3 个分解时间点 $(113$, 198 和 386 d)均存在显著的正相关关系，但在最后一个 时间点 (586 d)两者的显著性关系消失(图6(a)). 与碳元 素的格局相反，叶与细根间磷元素的残余率在第一个 分解时间点无显著关系，但在剩余的 3 个分解时间点均 

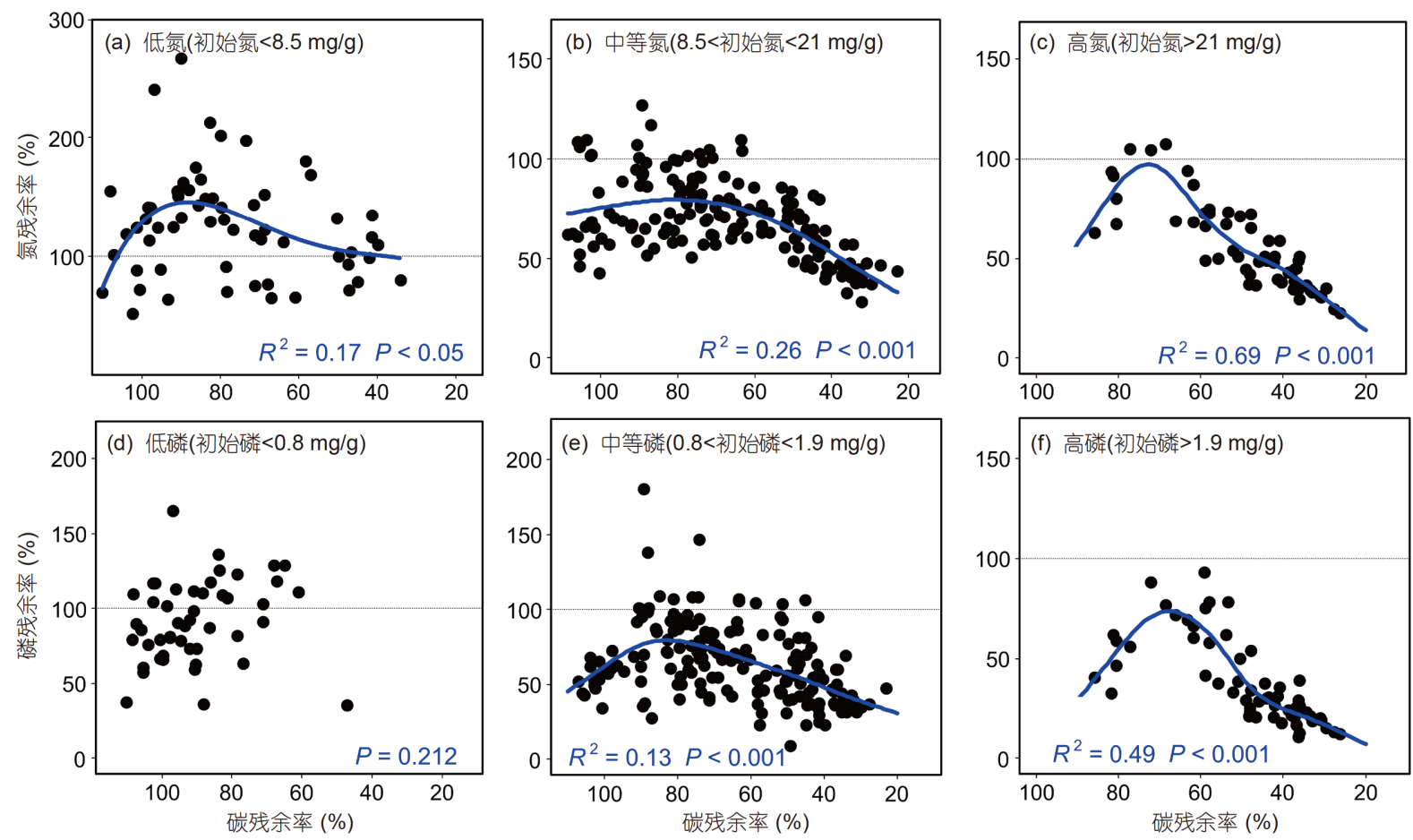

图 5 (网络版彩色) 荕类植物分解过程低、中和高初始氮数据组的氮残余率 $(\mathrm{a}) \sim(\mathrm{c})$, 低、中和高初始磷数据组的磷残余率 (d) $(\mathrm{f})$ 分别与碳残余 率间的相关性

Figure 5 (Color online) Fraction of original nitrogen (a)-(c) and phosphorus (d)-(f) remains as a function of the original carbon remains for the 12 fern species
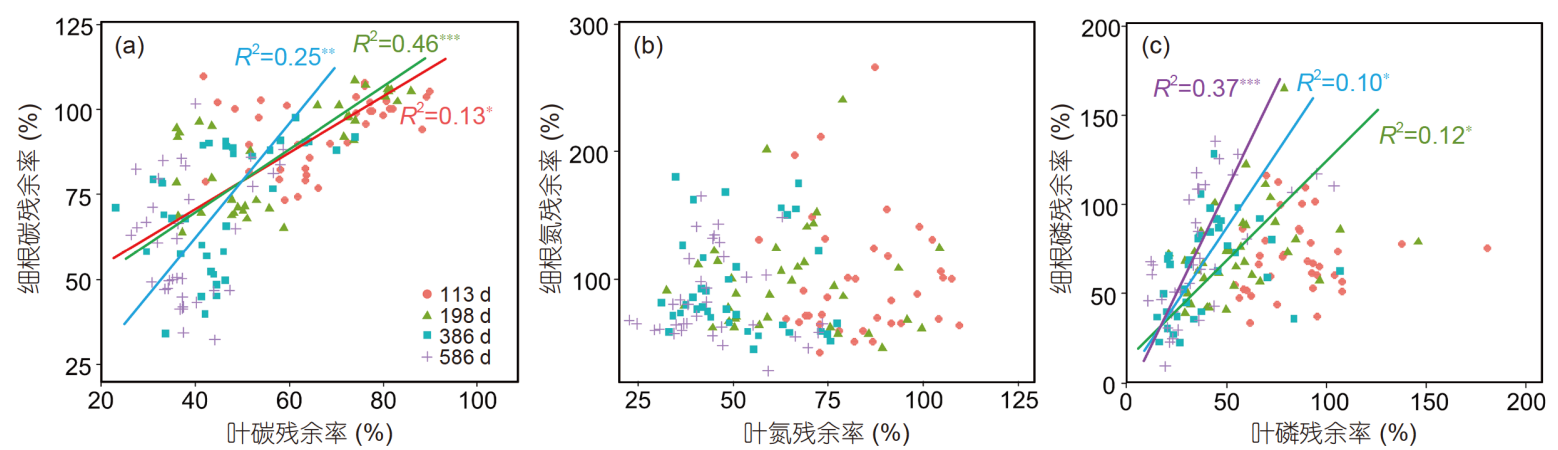

图 6 (网络版彩色)蒴类植物在分解 $113,198,386$ 和 $586 \mathrm{~d}$ 后叶和细根间碳(a)、氮(b)和磷(c)元素残余率的相关性. *, $P<0.05 ; * *, P<0.01 ; * * *$, $P<0.001$

Figure 6 (Color online) Covariation of carbon, nitrogen and phosphorus remains between leaves and roots after 113, 198, 386 and $586 \mathrm{~d}$ filed decomposition. *, $P<0.05 ; * *, P<0.01 ; * * *, P<0.001$

存在显著的正相关关系(图6(c)). 与碳磷的格局不同, 叶 与细根间氮元素的残留率在各分解时间点均无显著关 系(图6(b)).

\section{3 讨论}

调落物分解是生态系统物质循环的重要环节。尽 管烣类植物是热带和亚热带森林林下层物种多样性和
生物量的重要贡献者, 但我们对葭类植物在森林物质 循环中的重要性及其分解过程的元素释放规律的认识 还非常缺乏. 本研究对缙云山亚热带常绿阔叶林林下 常见偋类植物叶和细根分解过程的碳氮磷元素动态进 行了监测研究.

基质质量是影响调落物分解速率的三大重要因子 之一 ${ }^{[46]}$. 作为基质质量的重要指标, 调落物初始氮磷含 
量常是调落物分解快慢的限制性因子 ${ }^{[47,48]}$. 本研究中 偋类叶片的初始氮和磷含量均显著高于细根(表1), 这 意味着蒝类叶调落物能够为分解者提供更丰富的氮磷 元素从而促进微生物的生长, 预示着蕨类的叶将会以 更快的速率分解并释放养分. 的确, 我们发现除了一些 物种差异不显著外, 大多数物种经过 $568 \mathrm{~d}$ 的分解其叶 片的各指标残余率都显著低于细根, 即叶的分解速率 更快(图1)，这个结果总体上支持了我们的预测(1)。该 结果与在种子植物调落物分解中发现的格局类似，即 叶的分解速率要快于细根 ${ }^{[31,36 ~ 40]}$. 叶片与细根分解速 率的显著性差异意味着藏类叶和细根调落物的相对输 人量将是影响蒝类植物对森林碳和养分循环贡献强弱 的重要因素 ${ }^{[31]}$.

莿类碳元素与氮磷元素的释放表现出不同的格局 (图2 4). 碳元素的释放通常与调落物质量的减少呈线 性变化, 因此碳元素多表现为直接释放模式 ${ }^{[29]}$. 本研究 中除了部分难分解物种的细根在分解早期碳元素出现 微小上升外, 大部分叶和细根碳元素表现出直接释放 模式(图2). 从理论上讲, 碳元素的变化格局还会通过 影响化学计量比(碳氮比、碳磷比)从而间接调节调落 物分解过程中氮磷元素的释放 ${ }^{[28,29]}$. 我们针对偋类植 物的研究也表明了这一点. 随着分解的进行, 氮磷元素 出现直接释放以及富集-释放、富集-释放-富集、始终 富集等复杂模式(图3，4). 氮磷残余率与碳残余率的相 关性分析发现，在分解早期阶段无论初始氮磷含量的 高低, 氮磷元素残余率随碳的释放均是先增加(图 5), 这可能是因为在分解初期降雨的淋溶作用使可溶性氮 磷元素溶出 ${ }^{[49,50]}$ 导致残留在调落物上的氮磷元素不足 以满足后续生物分解者的需求，从而需要从周围环境 中吸收所需的氮磷元素 ${ }^{[26,28]}$; 而随着分解的继续进行, 氮磷元素残余率上升到一定程度后开始随着碳元素的 释放而减少(图5). 然而, 不同初始氮磷数据组间氮磷残 余率从增加到减少的转折点存在差异: 在低初始氮磷 的情况下，转折点的氮磷残留率在 $100 \%$ 以上，而中高 初始氮磷数据组转折点在 $100 \%$ 以下，这说明了初始氮 磷元素的含量与碳元素的含量一起影响了调落物分解 过程中氮磷元素的释放或富集. 这个结果支持了我们
的预测(2). 我们的研究结果表明, 生态化学计量学理 论同样适用于解释偋类植物分解中的元素释放动态, 这说明尽管蓱类植物与种子植物类群在进化历史上存 在一定的差异 ${ }^{[11,18]}$, 它们的调落物分解可能共享了土 壤中的微生物分解者.

植物经济谱理论认为植物的地上-地下的功能性状 存在协变性从而维持植物处于最佳的生长状态 ${ }^{[32]}$, 而 这些性状往往也可以很好地影响和预测植物调落物的 分解速率 ${ }^{[40,51 ~ 53]}$, 基于此推断植物叶片和细根的分解 速率应该也存在协变性 ${ }^{[32,40]}$. 然而, 对于该推论目前尚 存较大的争议, 已有的研究既有发现叶和细根分解速 率存在正相关关系 ${ }^{[34,38 \sim 40]}$, 也有发现两者为负相关或

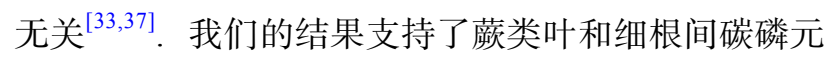
素的释放存在相关关系的预测(3). 此外, 我们发现蒴 类叶与细根碳元素的残留率仅在早期分解阶段存在显 著的正相关关系, 但在分解后期相关性消失(图6(a)). 这 个结果与 $\mathrm{Ma}$ 等人 ${ }^{[35]}$ 针对种子植物叶片和细根分解的 研究结果相似，他们发现两者的分解速率仅在分解的 前3个月表现出相关性，而在分解后期没有显著相关 性. 对于叶片和细根分解的养分动态, 分析发现氮元素 在任何一个分解阶段均不存在相关性, 而磷元素在分 解初期无相关性但在后续的分解阶段都表现出正相关 关系(图6(b), (c)). 这些结果说明, 叶片和细根分解间是 否存在协变关系受到分解阶段及研究对象 (不同化学 元素)的影响. 基于这些结果, 我们强调要充分认识蒝类 植物对生态系统碳和养分循环的影响必须同时对各器 官及不同元素开展量化研究.

\section{4 结论}

虽然䓲类植物与种子植物在解剖结构、生理特征

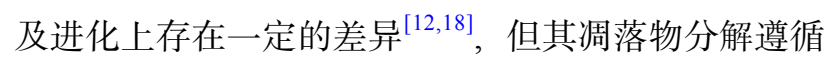
的规律与在种子植物中发现的规律较为类似: (1) 蒝类 叶分解速率快于细根; (2) 初始氮磷含量影响分解过程 中的氮磷元素释放格局; (3) 叶片和细根间元素释放的 协变关系因分解阶段及元素的不同而存在差异. 本研 究结果为进一步量化硕类植物对森林生态系统碳和养 分循环的重要性提供参考.

致谢感谢重庆师范大学何海教授帮忙鉴定茨类植物, 重庆大学庞梅和西南大学刘兰兰帮忙开展野外采样和实验室工作. 感 谢北京师范大学外文学院林敦来副教授对英文摘要提供修改建议. 


\section{参考文献}

1 Berg B, Mcclaugherty C. Plant Litter: Decomposition, Humus Formation, Carbon Sequestration. New York: Springer, 2003

2 Parton W, Silver W L, Burke I C, et al. Global-scale similarities in nitrogen release patterns during long-term decomposition. Science, 2007, 315: 361-364

3 Manzoni S, Jackson R B, Trofymow J A, et al. The global stoichiometry of litter nitrogen mineralization. Science, 2008, 321: 684-686

4 Handa I T, Aerts R, Berendse F, et al. Consequences of biodiversity loss for litter decomposition across biomes. Nature, 2014, 509: 218-221

5 Lin D, Pang M, Fanin N, et al. Fungi participate in driving home-field advantage of litter decomposition in a subtropical forest. Plant Soil, 2019, 434: $467-480$

6 Wang R H, Ge X M, Tang L Z. A review of diversity, biomass and nutrient effect of understory vegetation (in Chinese). Word Forestry Res, 2014, 27: 43-48 [王瑞华, 葛晓敏, 唐罗忠. 林下植被多样性、生物量及养分作用研究进展. 世界林业研究, 2014, 27: 43-48]

7 Nilsson M C, Wardle D A. Understory vegetation as a forest ecosystem driver: Evidence from the northern Swedish boreal forest. Front Ecol Environ, 2005, 3: 421-428

8 Gilliam F S. The ecological significance of the herbaceous layer in temperate forest ecosystems. BioScience, 2007, 57: 845-858

9 Brock J M R, Perry G L W, Burkhardt T, et al. Forest seedling community response to understorey filtering by tree ferns. J Veg Sci, 2018, 29: 887897

10 Royo A A, Carson W P. On the formation of dense understory layers in forests worldwide: Consequences and implications for forest dynamics, biodiversity, and succession. Can J For Res, 2006, 36: 1345-1362

11 Zhao J, Wan S, Li Z, et al. Dicranopteris-dominated understory as major driver of intensive forest ecosystem in humid subtropical and tropical region. Soil Biol Biochem, 2012, 49: 78-87

12 Mehltreter K, Walker L R, Sharpe J M. Fern Ecology. New York: Cambridge University Press, 2010

$13 \mathrm{Wu}$ J, Liu Z, Wang X, et al. Effects of understory removal and tree girdling on soil microbial community composition and litter decomposition in two Eucalyptus plantations in South China. Funct Ecol, 2011, 25: 921-931

14 Liu Y C, Liu Q J, Wang H Q, et al. Characteristices of biomass allocation of Dicranopteris dichotoma (in Chinese). Chin J Ecol, 2008, 27: 705-711 [刘迎春, 刘琪璟, 汪宏清, 等. 芒萁生物量分布特征. 生态学杂志, 2008, 27: 705-711]

15 Ma Z Q, Liu Q J, Xu W J, et al. A preliminary study on biomass of Woodwardia japonica community under a coniferous plantation in subtropical China (in Chinese). J Plant Ecol (Chin Ver), 2008, 32: 88-94 [马泽清, 刘琪璟, 徐雯佳, 等. 江西千烟洲人工针叶林下狗脊偋群落生物量. 植物 生态学报, 2008, 32: 88-94]

16 Lyu M, Xie J, Giardina C P, et al. Understory ferns alter soil carbon chemistry and increase carbon storage during reforestation with native pine on previously degraded sites. Soil Biol Biochem, 2019, 132: 80-92

17 Marsh A S, Arnone J A, Bormann B T, et al. The role of Equisetum in nutrient cycling in an Alaskan shrub wetland. J Ecol, 2000, 88: 999-1011

18 Page C N. Ecological strategies in fern evolution: A neopteridological overview. Rev Palaeobot Palynol, 2002, 119: 1-33

19 Karst A L, Lechowicz M J. Are correlations among foliar traits in ferns consistent with those in the seed plants? New Phytol, 2007, 173: 306-312

20 Han W, Fang J, Guo D, et al. Leaf nitrogen and phosphorus stoichiometry across 753 terrestrial plant species in China. New Phytol, 2005, 168: 377-385

21 Wardle D A, Bonner K I, Barker G M. Linkages between plant litter decomposition, litter quality, and vegetation responses to herbivores. Funct Ecol, 2002, 16: 585-595

22 Amatangelo K L, Vitousek P M. Contrasting predictors of fern versus angiosperm decomposition in a common garden. Biotropica, 2009, 41: 154161

23 Allison S D, Vitousek P M. Extracellular enzyme activities and carbon chemistry as drivers of tropical plant litter decomposition. Biotropica, 2004, 36: $285-296$

24 Shiels A B. Leaf litter decomposition and substrate chemistry of early successional species on landslides in Puerto Rico. Biotropica, 2006, 38: 348353

25 Bardgett R D. The Biology of Soil: A Community and Ecosystem Approach. New York: Oxford University Press, 2005

26 Mooshammer M, Wanek W, Zechmeister-Boltenstern S, et al. Stoichiometric imbalances between terrestrial decomposer communities and their resources: Mechanisms and implications of microbial adaptations to their resources. Front Microbiol, 2014, 5: 10

27 Sterner R W, Elser J J. Ecological Stoichiometry: The Biology of Elements from Molecules to the Biosphere. Princeton: Princeton University Press, 2002

28 Manzoni S, Trofymow J A, Jackson R B, et al. Stoichiometric controls on carbon, nitrogen, and phosphorus dynamics in decomposing litter. Ecol Monographs, 2010, 80: 89-106

29 Moore T R, Trofymow J A, Prescott C E, et al. Patterns of carbon, nitrogen and phosphorus dynamics in decomposing foliar litter in Canadian 
forests. Ecosystems, 2006, 9: 46-62

30 McCormack M L, Dickie I A, Eissenstat D M, et al. Redefining fine roots improves understanding of below-ground contributions to terrestrial biosphere processes. New Phytol, 2015, 207: 505-518

31 Freschet G T, Cornwell W K, Wardle D A, et al. Linking litter decomposition of above-and below-ground organs to plant-soil feedbacks worldwide. J Ecol, 2013, 101: 943-952

32 Reich B. The world-wide "ast-slow" plant economics spectrum: A traits manifesto. J Ecol, 2014, 102: 275-301

33 Hobbie S E, Oleksyn J, Eissenstat D M, et al. Fine root decomposition rates do not mirror those of leaf litter among temperate tree species. Oecologia, 2010, 162: 505-513

34 Fujii S, Makita N, Mori A S, et al. Plant species control and soil faunal involvement in the processes of above- and below-ground litter decomposition. Oikos, 2016, 125: 883-892

35 Ma C G, Xiong Y M, Li L, et al. Root and leaf decomposition become decoupled over time: Implications for below- and above-ground relationships. Funct Ecol, 2016, 30: 1239-1246

36 Sun T, Hobbie S E, Berg B, et al. Contrasting dynamics and trait controls in first-order root compared with leaf litter decomposition. Proc Natl Acad Sci USA, 2018, 115: 10392-10397

37 Vivanco L, Austin A T. Intrinsic effects of species on leaf litter and root decomposition: A comparison of temperate grasses from North and South America. Oecologia, 2006, 150: 97-107

38 Wang H, Liu S, Mo J. Correlation between leaf litter and fine root decomposition among subtropical tree species. Plant Soil, 2010, 335: 289-298

39 Birouste M, Kazakou E, Blanchard A, et al. Plant traits and decomposition: Are the relationships for roots comparable to those for leaves? Ann Bot, 2012, 109: 463-472

40 Freschet G T, Aerts R, Cornelissen J H C. A plant economics spectrum of litter decomposability. Funct Ecol, 2012, 26: 56-65

41 Song J H, Zhang H S, Sun C, et al. Canopy interception of different forest types in the Three Gorges Region of Yangtze River (in Chinese). Sci Soil Water Conserv, 2008, 6: 71-75 [宋吉红, 张洪江, 孙超, 等. 缙云山自然保护区不同森林类型林冠的截留作用. 中国水土保持科学, 2008, 6: $71-75]$

42 Cui Y Q, Du H, Deng H P, et al. Study on flora characteristics of pteridophyta in the Jinyun Mountains (in Chinese). J Southwest Univ, 2009, 31: 103-108 [崔亚琼, 杜红, 邓洪平, 等. 缙云山萨类植物区系特征研究. 西南大学学报, 2009, 31: 103-108]

43 Fujii S, Cornelissen J H C, Berg M P, et al. Tree leaf and root traits mediate soil faunal contribution to litter decomposition across an elevational gradient. Funct Ecol, 2018, 32: 840-852

44 Wood S N. Fast stable direct fitting and smoothness selection for generalized additive models. J Roy Stat Soc, 2010, 70: 495-518

45 Warton D I, Duursma R A, Falster D S, et al. Smatr 3- an R package for estimation and inference about allometric lines. Methods Ecol Evol, 2012, 3: $257-259$

46 Coûteaux M M, Bottner P, Berg B. Litter decomposition, climate and liter quality. Trends Ecol Evol, 1995, 10: 63-66

47 Hunt H W, Ingham E R, Coleman D C, et al. Nitrogen limitation of production and decomposition in prairie, mountain meadow, and pine forest. Ecology, 1988, 69: 1009-1016

48 Wieder W R, Cleveland C C, Townsend A R. Controls over leaf litter decomposition in wet tropical forests. Ecology, 2009, 90: 3333-3341

49 Schreeg L A, Mack M C, Turner B L. Nutrient-specific solubility patterns of leaf litter across 41 lowland tropical woody species. Ecology, 2013, 94: 94-105

50 Dou P P, Wang F, Ma Y, et al. Response of litter carbon, nitrogen and phosphorus to simulated leaching (in Chinese). Chin Sci Bull, 2018, 63: 3114-3123 [豆鹏鹏, 王芳, 马瑜, 等. 叶调落物碳、氮和磷元素对模拟淋溶的响应. 科学通报, 2018, 63: 3114-3123]

51 Santiago L S. Extending the leaf economics spectrum to decomposition: Evidence from a tropical forest. Ecology, 2007, 88: 1126-1131

52 Bakker M A, Carreño-Rocabado G, Poorter L. Leaf economics traits predict litter decomposition of tropical plants and differ among land use types. Funct Ecol, 2011, 25: 473-483

53 Lin D, Wang F, Fanin N, et al. Soil fauna promote litter decomposition but do not alter the relationship between leaf economics spectrum and litter decomposability. Soil Biol Biochem, 2019, doi.org/10.1016/j.soilbio.2019.107519 


\title{
Patterns of carbon, nitrogen and phosphorus dynamics during decomposition of fern leaf and fine root in a subtropical forest
}

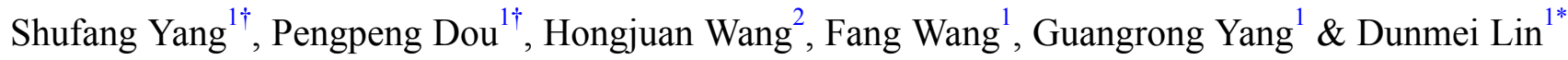 \\ ${ }^{1}$ Key Laboratory of the Three Gorges Reservoir Region's Eco-Environment, Ministry of Education, Chongqing University, Chongqing 400045, China; \\ ${ }^{2}$ Biotechnology Research Center, Chongqing Academy of Agricultural Sciences, Chongqing 401329, China \\ $\dagger$ These authors equally contributed to this work. \\ * Corresponding author, E-mail: lindunmei@cqu.edu.cn
}

Litter decomposition is a major driver of carbon and nutrient cycles and has major implications for carbon sequestration and plant nutrient availability in terrestrial ecosystems. The vast majority of litter decomposition studies have focused on the tree component in forest ecosystems, while little attention has been paid to understory components. Ferns, one ancient and diverse group of vascular plants, comprise a significant part of most forest understory layer. However, empirical study regarding decomposition rate and carbon, nitrogen and phosphorus release patterns of fern leaves and fine roots remains rare. Therefore, it is difficult to predict the ecological consequences of fern community changes resulting from forest management practices or other environmental changes.

In this study, we examined the patterns of mass loss and carbon, nitrogen, and phosphorus gain, retention or loss during the decomposition of leaves and fine roots $(<2 \mathrm{~mm})$ of 12 herbaceous fern species in a subtropical evergreen broad-leaved forest in Jinyun Mountain, Southwest China. We measured the initial carbon, nitrogen, and phosphorus concentrations of leaves and fine roots, and monitored the changes in carbon, nitrogen, and phosphorus after 113, 198, 386 and 586 day' decomposition in the field. We hypothesized that: $\left(\mathrm{H}_{1}\right)$ fern leaves would decompose faster than fine roots; $\left(\mathrm{H}_{2}\right)$ nitrogen and phosphorus release patterns would be affected by the initial tissue nitrogen and phosphorus concentrations; and $\left(\mathrm{H}_{3}\right)$ carbon, nitrogen and phosphorus release patterns of leaves and fine roots would coordinate across different fern species.

The results showed that: (1) Initial carbon concentration of fern leaves were similar to fine roots, while initial nitrogen and phosphorus concentrations of leaves were significantly higher than those of fine roots. Litter mass, carbon, nitrogen and phosphorus remains of fern leaves were generally significantly lower than those of fine roots, supporting our first hypothesis $\left(\mathrm{H}_{1}\right)$. (2) Carbon, nitrogen and phosphorus of fern leaves and roots exhibited different loss patterns. Leaf carbon, nitrogen and phosphorus generally directly lost during the entire decomposition process. By contrast, carbon, nitrogen and phosphorus of fine roots showed more complicated patterns such as direct loss, gain-loss, gain-loss-gain and always gain. In support of our second hypothesis $\left(\mathrm{H}_{2}\right)$, we found that nitrogen or phosphorus release patterns were influenced by the initial tissue nitrogen or phosphorus concentrations, respectively. (3) Carbon remains between leaves and fine roots were significantly correlated after 113, 198 and 386 day' decomposition in the field, but not after $586 \mathrm{~d}$. Phosphorus remains between leaves and fine roots were significantly correlated after 198, 386 and 586 day' decomposition in the field, but not after $113 \mathrm{~d}$. By contrast, there was no significant relationship of the nitrogen remains between leaves and fine roots during the entire decomposition process. Thus, our third hypothesis $\left(\mathrm{H}_{3}\right)$ was only partly supported. This result suggests that the above- and below-ground covariation patterns depend on specific element and the duration of decomposition. Our study improves our understanding of how fern species influence the carbon and nutrient cycling in subtropical forest.

fern, leaf decomposition, fine root decomposition, nutrient release, stoichiometry, above- and below-ground covariation

doi: 10.1360/TB-2019-0002 